\title{
Early results of pediatric appendicitis after adoption of diagnosis-related group-based payment system in South Korea
}

This article was published in the following Dove Press journal:

Journal of Multidisciplinary Healthcare

24 November 2015

Number of times this article has been viewed

\section{Suk-Bae Moon}

Department of Surgery, Kangwon National University Hospital, Kangwon National School of Medicine, Kangwon National University, Chuncheon, South Korea

Correspondence: Suk-Bae Moon Department of Surgery, Kangwon National University Hospital, Kangwon National School of Medicine, Kangwon National University, Chuncheon 200-722, South Korea

Tel +82 332589209

Fax +82332582169

Email sukbae75.moon@gmail.com
Purpose: As an alternative to the existing fee-for-service (FFS) system, a diagnosis-related group (DRG)-based payment system has been suggested. The aim of this study was to investigate the early results of pediatric appendicitis treatment under the DRG system, focusing on health care expenditure and quality of health care services.

Patients and methods: The medical records of 60 patients, 30 patients before (FFS group), and 30 patients after adoption of the DRG system (DRG), were reviewed retrospectively.

Results: Mean hospital stay was shortened, but the complication and readmission rates did not worsen in the DRG. Overall health care expenditure and self-payment decreased from Korean Won (KRW) 2,499,935 and KRW 985,540, respectively, in the FFS group to KRW 2,386,552 and KRW 492,920, respectively, in the DRG. The insurer's payment increased from KRW 1,514,395 in the FFS group to KRW 1,893,632 in the DRG. For patients in the DRG, calculation by the DRG system yielded greater overall expenditure (KRW 2,020,209 vs KRW 2,386,552) but lower self-payment (KRW 577,803 vs KRW 492,920) than calculation by the FFS system.

Conclusion: The DRG system worked well in pediatric patients with acute appendicitis in terms of cost-effectiveness over the short term. The gradual burden on the national health insurance fund should be taken into consideration.

Keywords: appendicitis, child, fee-for-service plans, diagnosis-related groups, quality of health care, health care expenditures

\section{Introduction}

Since the introduction of national health insurance in 1977, the reimbursement system for medical services has been based on a fee-for-service (FFS) system in South Korea. The FFS system is a kind of retrospective reimbursement system in which health care providers receive payment for each service rendered. Due to the relatively low fees compared to the prime cost of each service, the FFS system in South Korea has been accused of adding to national health care expenditure (HE) by excessively increasing the volume of health care services. ${ }^{1}$ In 1994, the diagnosis-related group (DRG)-based payment system was suggested as an alternative method to overcome the shortcomings of the FFS system and the former has undergone several revisions and a pilot project since that time. The DRG-based payment system is a type of case-based, prospective payment system under which payment is based on a particular diagnosis the amount for which is already determined. The ultimate goal of the DRG-based payment system is to lower the overall $\mathrm{HE}$ while maintaining the quality of health care services. However, one of the major concerns of health care providers regarding the DRG-based payment system has been the underlying risk of downgrading the quality 
of health care services in order to save prime cost and gain more profit. Moreover, there have been conflicting results on the total amount of HE before and after adoption of the pilot project of the DRG-based payment system. ${ }^{2}$

A DRG-based payment system that covers seven disease categories has been mandatory for every class of hospital in South Korea since July 2013. By focusing on HE and quality of health care, the aim of this study is to investigate the early results of pediatric appendicitis treatment under the DRG-based payment system (hereafter referred to as DRG system) and compare its cost-effectiveness with that of the FFS system.

\section{Patients and methods \\ Patients}

Between January 1, 2013, and June 30, 2013, 30 pediatric patients, aged 18 years or younger, underwent appendectomy under the FFS system (FFS group). After that, under the DRG system, 30 consecutive patients underwent appendectomy by a modified surgical technique and were cared for under the respective clinical pathway (DRG). The medical records of these 60 patients were reviewed retrospectively. Data collected are as follows: age, sex, diagnostic imaging modality (computed tomography vs ultrasonography), postoperative diagnosis (simple vs complicated appendicitis), operation method (open vs laparoscopic appendectomy), operation time, hospital stay, postoperative complications, readmission rate, DRG code, overall expenditure, self-payment, and insurer's payment of the expenditure.

\section{DRG coding}

The DRG code for the DRG was created according to the guidelines provided by the Health Insurance Review and Assessment Service (HIRA). ${ }^{3}$ The DRG code for acute appendicitis is composed of six digits, such as G08 $x 0 y$, where $x$ represents the disease category (complicated appendicitis $[x=1]$ or simple appendicitis $[x=2]$ treated by open appendectomy; vs complicated appendicitis $[x=3]$ or simple appendicitis $[x=4]$ treated by laparoscopic appendectomy) and $y$ represents complications or comorbidities (no $[y=0]$, moderate $[y=1]$, and severe $[y=2]$ complications or comorbidities). For comparison between groups according to the same code, patients in the FFS group were retrospectively coded according to the same guidelines. Complicated appendicitis was defined as a gangrenous or perforated appendicitis according to the intraoperative findings. National average hospital stay according to the DRG code was obtained from the database of HIRA.

\section{General laparoscopic technique, instruments, and disposable materials}

A laparoscopic approach was undertaken throughout the study period, except in some cases, in which a thin abdominal wall and a small body cavity made open appendectomy a more feasible procedure. All the operations were performed by a single surgeon (MSB). Laparoscopic techniques were identical before and after adoption of DRG except for the manner used to ligate the appendicular artery and appendicular base (described in detail in the section "Ligation of appendicular vessels and appendicular base before and after adoption of DRG"). Under general anesthesia, an $11 \mathrm{~mm}$ VersaStep trocar (United States Surgical, Norwalk, CT, USA) was placed transumbilically, and a $5 \mathrm{~mm}$ 30-degree laparoscope was inserted. Under direct vision, $5 \mathrm{~mm}$ VersaStep trocars were inserted in the suprapubic area and anti-McBurney's point, respectively. In patients with body weight $<40 \mathrm{~kg}$, a $3 \mathrm{~mm}$ trocar (Karl Storz Endoskope, Tuttlingen, Germany) was used instead for the suprapubic area. After ligating the appendicular vessels, the inflamed appendix was resected. The specimen was then removed with a retrieval bag (LapBag; Sejong Medical Co, Paju, Kyoungki, South Korea) through the umbilical wound. The umbilical wound was closed in two layers: fascia with 2-0 Vicryl (Ethicon, Somerville, NJ, USA) and dermis with 4-0 Vicryl. The remaining wounds were closed in two layers: dermis with 4-0 Vicryl and skin with Dermabond (Ethicon). Aseptic dressing was applied only to the umbilical wound (Mepilex Border; Mölnlycke Health Care, Gothenburg, Sweden).

\section{Ligation of appendicular vessels and appendicular base before and after adoption of DRG}

Before adoption of the DRG system, the mesoappendix was resected with a LigaSure vessel-sealing system (Valleylab, Boulder, CO, USA), and the appendicular base was doubly ligated with Vicryl Endoloop ligature (Ethicon). After adoption of the DRG system, however, to lower prime cost, the appendicular vessels were ligated with double Hem-olok ${ }^{\circledR}$ clips (Weck Closure Systems, Research Triangle Park, Durham, NC, USA). The proximal base of the appendix was closed using a single Hem-o-lok clip.

\section{Clinical pathway for postoperative care}

For simple appendicitis, which was defined as appendicitis without evidences of perforation, gangrene, or abscess formation, postoperative care was provided according to the clinical pathway. A synopsis of the protocol is as follows: 
patients received $20 \mathrm{mg} / \mathrm{kg}$ of cefotetan preoperatively (Yamatetan; JEIL Pharmaceutical Co, Seoul, South Korea). It was continued through postoperative day 1 , dosing every 12 hours, and discontinued on postoperative day 2. Patients received preoperative laboratory workup, and no additional laboratory tests were obtained until postoperative day 2, when a complete blood cell count was obtained to confirm normalization of the white blood cell count. Diet was resumed on the morning of postoperative day 1 and advanced, while intravenous fluids were tapered. Patients were discharged on postoperative day 2 without any oral medications. Complicated appendicitis was excluded from the clinical pathway and cared for individually according to the clinical situation.

\section{Calculation of the HE}

Under the FFS system, the overall cost was determined by summation of the costs of individual medical services provided, and the overall cost was then divided into self-payment and insurer's payment according to the proportion set by the government. Under the DRG system, the overall cost was determined by the patients' DRG codes, and the overall cost was divided into self-payment and insurer's payment according to the proportion set by the government.

\section{Outcome measurement}

Quality of health care was measured by mean hospital stay, postoperative complication rate, and readmission rate and then compared between the two groups. Mean hospital stay was compared to the national average for the most frequently registered DRG code during the study period. Mean HE measured in each patient group by either the FFS or the DRG system was denoted as HE-"PATIENT GROUP" "PAYMENT SYSTEM". Mean HE measured in each patient group according to the payment system to which the patients belonged (HE-DRG ${ }_{\text {DRG }}$ and $\mathrm{HE}-\mathrm{FFS}_{\mathrm{FFS}}$ ) was then divided into self-payment and insurer's payment and compared between the two groups. $\mathrm{HE}^{-D R G_{\mathrm{DRG}}}$ was converted according to the FFS system $\left(\mathrm{HE}-\mathrm{DRG}_{\mathrm{FFS}}\right.$ ) and compared with $\mathrm{HE}_{-\mathrm{DRG}} \mathrm{DRG}_{\mathrm{D}}$ to measure the changes in $\mathrm{HE}$ in the DRG according to the payment system. HEs were compared between the two groups according to the DRG code. Because the prime cost consumed per patient would be more proportional to the cost calculated by the FFS system, HE-DRG $\mathrm{FFS}_{\mathrm{F}}$ was compared to HE-FFS $\mathrm{FFs}_{\text {. }}$.

\section{Statistical analysis}

SPSS package (Version 16.0, SPSS Inc., Chicago, IL, USA) was used to perform statistical analyses. Continuous variables were compared using Student's $t$-test, and dichotomous variables were compared with chi-square test. A $P$-value $<0.05$ was considered statistically significant.

\section{Ethics statement}

This study was approved by the Kangwon National University Hospital Institutional Review Board on June 30, 2014 (KWNUH 2014-05-009). This review was conducted in accordance with all guidelines stipulated by the Declaration of Helsinki. Because no interventional experimentation whatsoever was involved and the study was retrospective, the Kangwon National University Hospital Institutional Review Board decided that written patient consent was not required.

\section{Results}

\section{Patient demographics}

There were no significant statistical differences with regard to the patients' age, sex ratio, operation method, and operation time between the two groups. Ultrasonography confirmed the diagnosis in two patients in the FFS group, while computed tomography confirmed the diagnosis in every patient in the DRG. The most frequent DRG code was G08400 in both groups, followed by G08300 and G08200. Detailed patient demographics are listed in Table 1.

\section{Quality of health care}

Mean hospital stay decreased slightly after adoption of the DRG system, but the change was not statistically significant.

Table I Patient demographics

\begin{tabular}{|c|c|c|c|}
\hline Characteristics & FFS group $(n=30)$ & DRG $(n=30)$ & $P$-value \\
\hline $\begin{array}{l}\text { Mean age (range), } \\
\text { years }\end{array}$ & $13.4(6-18)$ & $12.9(5-18)$ & 0.68 \\
\hline Sex ratio (M:F) & $19: 11$ & $18: 12$ & 1 \\
\hline Operation method & $28 \mathrm{LA}, 2 \mathrm{OA}$ & $28 \mathrm{LA}, 2 \mathrm{OA}$ & 1 \\
\hline $\begin{array}{l}\text { Operation time } \\
\text { (range), minutes }\end{array}$ & $35.2(20-70)$ & $39.1(30-60)$ & 0.09 \\
\hline Imaging modality & 28 CT, 2 USG & $30 \mathrm{CT}$ & 0.49 \\
\hline $\begin{array}{l}\text { Postoperative } \\
\text { diagnosis }\end{array}$ & $26 \mathrm{SA}, 4 \mathrm{CA}$ & $27 \mathrm{SA}, 3 \mathrm{CA}$ & \\
\hline DRG code $(n)$ & & & \\
\hline G08400 & 24 & 25 & \\
\hline G08300 & 3 & 2 & \\
\hline G0830I & 0 & 1 & \\
\hline G08302 & 1 & 0 & \\
\hline G08200 & 2 & 2 & \\
\hline
\end{tabular}

Abbreviations: CA, complicated appendicitis; CT, computed tomography; DRG, diagnosis-related group; F, female; FFS, fee-for-service; LA, laparoscopic appendectomy; M, male; OA, open appendectomy; SA, simple appendicitis; USG, ultrasonography. 
Table 2 Quality of health care before and after adoption of the DRG system

\begin{tabular}{llll}
\hline & FFS group $(\mathbf{n}=\mathbf{3 0})$ & DRG $(\mathbf{n}=\mathbf{3 0})$ & P-value \\
\hline $\begin{array}{l}\text { Mean hospital stay } \\
\text { Postoperative }\end{array}$ & I.6 days (3-13 days) & 3.5 days (3-8 days) & 0.80 \\
complications & & I (ileus) & $\mathrm{I}$ \\
Readmission & 0 & 0 & $\mathrm{I}$ \\
\hline
\end{tabular}

Abbreviations: DRG, diagnosis-related group; FFS, fee-for-service.

Complication rate and readmission rate for complications showed no significant difference before and after adoption of the DRG system (Table 2). For the most frequent G08400 code, the national average hospital stay was 5.13 days, which was longer than that of our study group (3.28 days, $P=0.00)$.

\section{Health care expenditure}

After calculating the mean expenditure according to the payment system to which each patient belonged, overall expenditures and self-payment decreased from Korean Won (KRW) 2,499,935 and KRW 985,540, respectively, before adoption of the DRG system (HE-FFS ${ }_{\mathrm{FFS}}$ ) to KRW 2,386,552 and KRW 492,920, respectively, after adoption of the DRG system $\left(H E-D R G_{\text {DRG }}\right.$ ), while the insurer's payment increased from KRW 1,514,395 before adoption of the DRG system to KRW 1,893,632 after adoption of the DRG system (Figure 1). For the most frequent DRG code (G08400), HE slightly decreased in the DRG, whereas HE increased in the DRG with G08300 (Figure 2). One patient in the FFS group developed an abscess

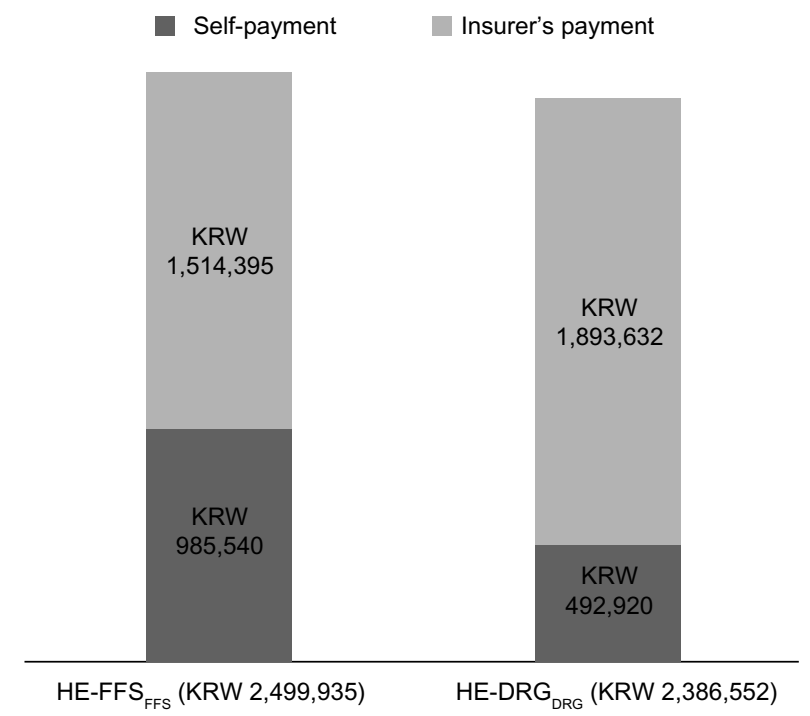

Figure I Health care expenditures in both groups.

Note: Overall expenditure and self-payment decreased in the DRG.

Abbreviations: DRG, diagnosis-related group; FFS, fee-for-service; $H E$, health care expenditure; $H E-D R G_{D R G}$, HE in DRG group measured by DRG system; HE-DRG ${ }_{F F S}$, HE in DRG group measured by FFS system; KRW, Korean Won. postoperatively and required percutaneous drainage with a longer hospital stay, consuming an excessive cost of KRW $5,094,818$ (Figure 2). For patients in the DRG, $H E-D R G_{D R G}$ yielded a greater total expenditure (KRW 2,020,209 vs KRW 2,386,552) but smaller self-payment (KRW 577,803 vs KRW

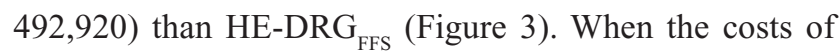
both groups were calculated using the same FFS system, HE-FFS $_{\mathrm{FFS}}$ was greater than HE-DRG $\mathrm{FFS}_{\mathrm{FR}}$ (KR,499,935 vs KRW 2,020,209).

\section{Discussion}

Although limited to pediatric appendicitis, this study attempted to determine whether the DRG system meets its own objectives, by comparing the HE and quality of health care before and after adoption of the DRG system. Under the DRG system, there was a tendency for the readmission rate to increase, ${ }^{4-6}$ hospital stay to decrease, and admission rate to decrease. ${ }^{7-9}$ The South Korean DRG pilot project launched in 1997 demonstrated positive effects, such as reduction in hospital stay, medical expenses, average number of tests, and use of antibiotics without having a negative effect on quality of care. ${ }^{10}$ In this study, hospital stay was shortened in the DRG, while the complication and readmission rates did not worsen. For the most common DRG code, our average hospital stay was shorter than the national average. Using an evidence-based clinical pathway for pediatric appendicitis has been proven to decrease resource utilization and permit early discharge. ${ }^{11,12}$ We speculate that our results might be the consequence of a well-organized clinical pathway for postoperative care that restricts hospital stay to 3 days and minimizes resource utilization in cases of simple appendicitis. According to the HIRA database, the national average hospital stay for the category covering the most severe cases of appendicitis was 8.61 days (range: $3-17$ days). Although we did not follow a clinical pathway in cases of complicated appendicitis and acquired similar results compared to the national average, a well-designed clinical pathway for complicated appendicitis based on current practice would produce better results in the future.

When compared against the same patient group, charging by the DRG system yields more expenditure than by the FFS system, implying an increased economic reward in the DRG system for providing the insurance-covered medical services. Because one of the persistent downsides of the South Korean FFS system has been its relatively low fees for each medical service compared to the prime cost, it would be a positive aspect if the current DRG system were to increase the economic reward for the insurance-covered medical 
FFS group $\quad$ DRG group

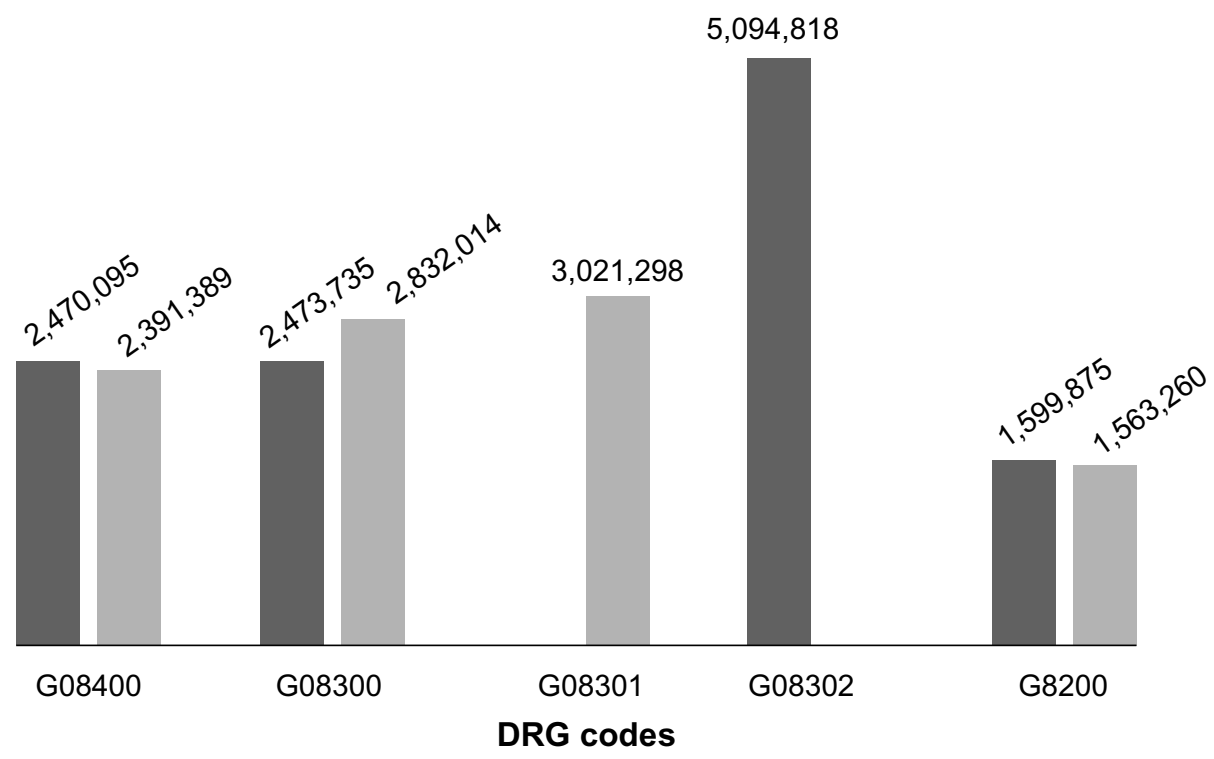

Figure 2 Health care expenditures stratified by the DRG code.

Note: One patient in the FFS group consumed an excessive cost of KRW 5,094,8I8 due to postoperative complication. Values are given in KRW. Abbreviations: DRG, diagnosis-related group; FFS, fee-for-service; KRW, Korean Won.

services. When the costs of two groups were calculated by the FFS payment system, HE-DRG ${ }_{\mathrm{FFS}}$ was smaller than HEFFS $_{\mathrm{FFS}}$, meaning a reduction of prime costs in the DRG as expensive devices and materials were no longer used during each operation. In cases of typical, uncomplicated appendicitis, health care providers would benefit from increased economic reward with a reduction of prime cost under the DRG system.

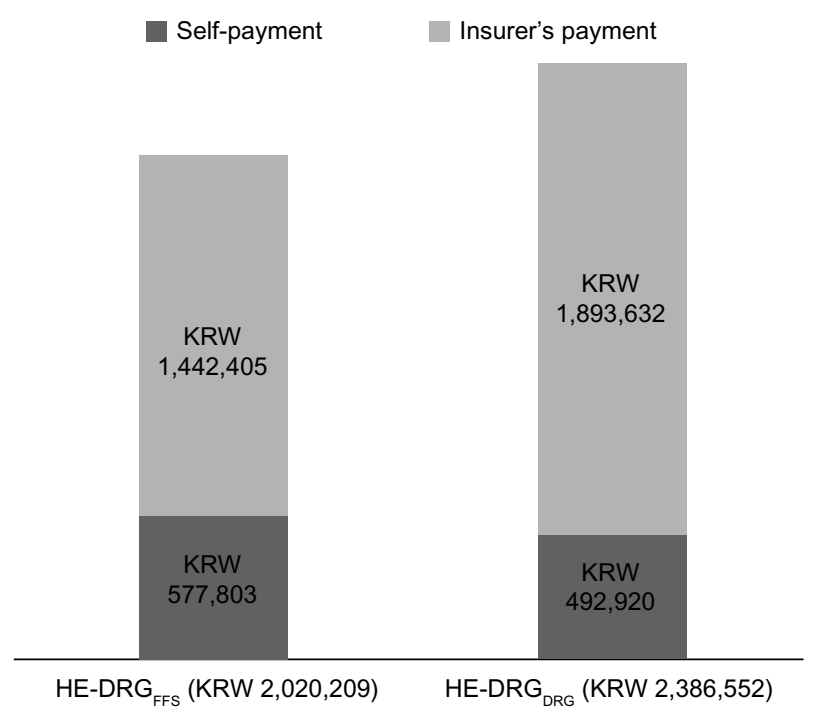

Figure 3 For the DRG patients, calculation by the DRG system yielded more overall expenditure but less self-payment.

Abbreviations: DRG, diagnosis-related group; FFS, fee-for-service; $\mathrm{HE}$, health care expenditure; HE-DRG ${ }_{D R G}$, HE in DRG group measured by DRG system; HE-DRG FFs' $_{\text {, }}$ HE in DRG group measured by FFS system; KRW, Korean Won.
As the DRG system originally intended, overall HE decreased in the DRG, although the decrease was not so pronounced in this study. It would seem contradictory to be able to reduce the overall HE with increased economic reward for the insurance-covered services under the DRG system. Our speculation is as follows: in complicated appendicitis, unpredictable events such as ileus or abscess could take place at any time during the postoperative period, and costs exceeding the DRG coverage limit might be required. If calculated using the DRG fee calculator of HIRA (http:// www.hira.or.kr), the maximal coverage limit of the current DRG system for the patient who developed a postoperative abscess in the FFS group was KRW 3,438,530, a figure that was far below the actual cost. The DRG system subdivided the costs according to the combination of 12 major categories of appendicitis (DRG code G08100-G08402) and hospital stays of variable lengths (1-30 days), but covering all events with limited disease categories and expenses is sometimes impossible. Although one of the major objectives of the DRG system is to maintain HE within a proper limit, the objective should be pursued in such a way as to suppress unnecessary, excessive services that could occur under the FFS system, not in a way that overlooks compensation for essential medical services. The current system compels one to follow the DRG system, even in cases for which the system cannot compensate. It might be an easy way to control HE, but it also leads to financial loss for health care providers in some 
cases, and such a policy could not be justified. There should be a compensatory system for such situations.

Patients' self-payments decreased in the DRG, and this is one of the advantages of the DRG system that the South Korean government has promoted thus far. In conjunction with the elevated economic rewards, it would therefore seem to be a "win-win" situation, both for health care providers and patients. However, decrease in self-payment implies an increase in the insurer's payment, which will eventually lead to a further burden on national health insurance funds. Substantial increases in health insurance contributions seem to be inevitable in the near future if this condition persists. Inevitably, South Korea will need to raise insurance contributions to respond to sharply increasing medical expenses due to having the highest proportion of an aging population in the world, ${ }^{13}$ and an increase in health insurance contributions following the DRG system should be considered within the same context.

Before adoption of the DRG system, we routinely used LigaSure and Vicryl Endoloop ligature. These materials were not covered by insurance, rather expensive, and charged solely to patients. After adoption of the DRG system, we moved to using Hem-o-lok clips instead to save prime costs and achieved similar postoperative outcomes. We speculate that this shows two sides of the same coin. On the positive side, it shows that appendectomy can be performed safely without increasing the prime costs. Hem-o-lok clips have been proven to have the advantages of easy application and low cost compared with the endoloop or stapler in laparoscopic appendectomy. ${ }^{14,15}$ However, on the negative side, it would be a "back to the past". Certainly, LigaSure or ultrasonic shear such as a Harmonic Scalpel (Ethicon EndoSurgery, Cincinnati, OH, USA) is a technically advanced device compared to the Hem-o-lok system and has a wider range of indications that would not be amenable to plastic clips. Such devices could facilitate the dissection of mesoappendix in complicated appendicitis and safely shorten the operation time. ${ }^{16}$ However, the current DRG system does not consider advanced technology and instruments, and such an adherence to cheap, old-fashioned techniques and devices would eventually have adverse effects on patients' safety.

In the DRG, one patient developed appendicitis after surgery for osmidrosis in the Department of Plastic Surgery. The patient underwent appendectomy 1 day after osmidrosis surgery, and fees related to the treatment of osmidrosis could not be claimed. The current DRG system dictates that there be at least 6 days' interval between the preceding treatment and the DRG system-covered treatment to claim separate fees.
In this case, however, it would be practically impossible to delay the appendectomy by 6 days to claim separate fees. This case shows another weak point of the current DRG system, which rejects reasonable compensation following any other combined treatment. Under these circumstances, medical practitioners could be motivated to avoid patients who need concurrent therapy that is not covered by the DRG system, and this would eventually lead to patients' dissatisfaction.

\section{Conclusion}

In conclusion, the DRG system worked well in pediatric patients with acute appendicitis in terms of cost-effectiveness over the short term. The burden to patients has been relieved by a lowered self-payment, the hospital stay has been shortened, and the complication rate has remained unchanged. However, the gradual burden to the national health insurance fund would contraindicate the goals of the current DRG system. Repair of the aforementioned institutional defects, compensation for essential medical services, and consideration of up-to-date technology should supplement the revised system.

\section{Disclosure}

The author reports no conflicts of interest in this work.

\section{References}

1. Kim YK. Forecasting the future reimbursement system of Korean National Health Insurance: a contemplation focusing on global budget and Neo-KDRG-based payment systems. J Korean Med Sci. 2012;27(Suppl):S25-S32.

2. Korean Health Insurance Review and Assessment Service. An Evaluation on Neo-KDRG-Based Payment System Pilot Project. Seoul: Korean Health Insurance Review and Assessment Service; 2010.

3. Health Insurance Review and Assessment Service. DRG Practice Manual. Seoul: Health Insurance Review and Assessment Service; 2013.

4. Leibson CL, Naessens JM, Campion ME, Krishan I, Ballard DJ. Trends in elderly hospitalization and readmission rates for a geographically defined population: pre- and post-prospective payment. J Am Geriatr Soc. 1991;39:895-904.

5. Brizioli E, Fraticelli A, Marcobelli A, Paciaroni E. Hospital payment system based on diagnosis related groups in Italy: early effects on elderly patients with heart failure. Arch Gerontol Geriatr. 1996;23:347-355.

6. Hamada H, Sekimoto M, Imanaka Y. Effects of the per diem prospective payment system with DRG-like grouping system (DPC/PDPS) on resource usage and healthcare quality in Japan. Health Policy. 2012;107:194-201.

7. Schwartz WB, Mendelson DN. Hospital cost containment in the 1980s. Hard lessons learned and prospects for the 1990s. N Engl J Med. 1991;324:1037-1042.

8. Louis DZ, Yuen EJ, Braga M, et al. Impact of a DRG-based hospital financing system on quality and outcomes of care in Italy. Health Serv Res. 1999;34:405-415.

9. Busato A, von Below G. The implementation of DRG-based hospital reimbursement in Switzerland: a population-based perspective. Health Res Policy Syst. 2010;8:31.

10. Kwon S. Payment system reform for health care providers in Korea. Health Policy Plan. 2003;18:84-93. 
11. Bensard DD, Hendrickson RJ, Fyffe CJ, Careskey JM, Azizkhan RG. Early discharge following laparoscopic appendectomy in children utilizing an evidence-based clinical pathway. J Laparoendosc Adv Surg Tech A. 2009;19(Suppl 1):S81-S86.

12. Knott EM, Gasior AC, Ostlie DJ, Holcomb GW 3rd, St Peter SD. Decreased resource utilization since initiation of institutional clinical pathway for care of children with perforated appendicitis. $J$ Pediatr Surg. 2013;48:1395-1398.

13. Kim KS, Lee YJ. Developments and general features of National Health Insurance in Korea. Soc Work Public Health. 2010;25:142-157.
14. Delibegović S, Matović E. Hem-o-lok plastic clips in securing of the base of the appendix during laparoscopic appendectomy. Surg Endosc. 2009;23:2851-2854.

15. Delibegović $\mathrm{S}$. The use of a single Hem-o-lok clip in securing the base of the appendix during laparoscopic appendectomy. $J$ Laparoendosc Adv Surg Tech A. 2012;22:85-87.

16. Aydogan F, Saribeyoglu K, Simsek O, et al. Comparison of the electrothermal vessel-sealing system versus endoclip in laparoscopic appendectomy. J Laparoendosc Adv Surg Tech A. 2009;19:375-378.

\section{Publish your work in this journal}

The Journal of Multidisciplinary Healthcare is an international, peerreviewed open-access journal that aims to represent and publish research in healthcare areas delivered by practitioners of different disciplines. This includes studies and reviews conducted by multidisciplinary teams as well as research which evaluates the results or conduct of such teams or health- care processes in general. The journal covers a wide range of areas and welcomes submissions from practitioners at all levels, from all over the world The manuscript management system is completely online and includes a very quick and fair peer-review system. Visit http://www.dovepress.com/ testimonials.php to read real quotes from published authors.

Submit your manuscript here: http://www.dovepress.com/journal-of-multidisciplinary-healthcare-journal 